\title{
Coronal oscillation above a supergranular cell of the quiet Sun chromospheric network?
}

\author{
C. Gontikakis ${ }^{1}$, H. Peter ${ }^{2}$, and H. C. Dara ${ }^{1}$ \\ 1 Academy of Athens, Research Center of Astronomy and Applied Mathematics, Soranou Efessiou 4, 11527 Athens, Greece \\ e-mail: [cgontik; edara]@cc.uoa.gr \\ 2 Kiepenheuer-Institut für Sonnenphysik, Schöneckstr. 6, 79104 Freiburg, Germany \\ e-mail: peter@kis.uni-freiburg.de
}

Received 22 February 2005 / Accepted 24 June 2005

\section{ABSTRACT}

We have detected an oscillation in the low corona, using a raster of the SUMER EUV spectrograph in the Ne VIII, $770 \AA$ Aine formed at about $700000 \mathrm{~K}$. The oscillation was found in the Ne VIII Doppler map above the interior of a supergranular cell of the chromospheric network in the quiet Sun, while it is absent in line radiance. The photospheric magnetic field, extrapolated to coronal levels, was used to relate this phenomenon to the magnetic structure. This oscillation phenomenon, reported here for the first time, seems to occur only above the darkest cells of the chromospheric network. We interpret our findings as a collective non-compressible oscillation of the corona above the whole cell interior. This oscillation may originate at the chromosphere and the driving wave may undergo a mode conversion at the top chromosphere, where the magnetic pressure equals the gas pressure. Our interpretation cannot be definitive and should be verified with more data.

Key words. Sun: UV radiation - Sun: corona

\section{Introduction}

The analysis of oscillations in the solar atmosphere can be a useful tool to understand its properties. Carlsson \& Stein's hydrodynamic simulations $(1995,1997)$ showed the coupling between the photospheric acoustic oscillations and the bright grains observed in the internetwork (non-magnetic) chromosphere.

Space-born solar telescopes, after the mid 90ies, provided observations with time series of UV lines and continua, emitted by the chromosphere and transition region, with a good time resolution. These observations allowed a deeper study of the mentioned atmospheric layers. The combination of MDI magnetograms and Doppler maps with TRACE continuum images and SUMER spectra provided the possibility to study the atmosphere from the photosphere to the transition region (e.g. Judge et al. 2001). It was realized that the power of the acoustic waves in the internetwork, responsible for the appearance of the Ca II K grains (Carlsson \& Stein 1995, 1997) decreases with altitude when reaching the transition region (Judge et al. 1997; Gouttebroze et al. 1999). However, wave power does not totally disappear, as SUMER Doppler maps in transition region lines indicate the presence of upward wave propagation (Carlsson et al. 1997; Judge et al. 1997; Wikstøl et al. 2000). These oscillations, apparent more in Doppler shift than radiance transition region time series, preceeded the ones recorded in the low chromosphere (Wikstøl et al. 2000).
The magnetic field strength and topology have an influence on the wave modulation even in the internetwork which, at first order, can be considered as non magnetic. The wave power is depleted near the magnetic network elements, giving rise to the so called magnetic shadows (Judge et al. 2001). Moreover, in the internetwork the strongest wave power is measured at locations where the height of plasma $\beta=1$ is found at higher altitudes in the solar atmosphere (McIntosh et al. 2001). $\beta$ is defined as the ratio of the gas to the magnetic pressure.

Most studies of oscillations of the quiet Sun focus at the frequency range of 3-10 $\mathrm{mHz}$. There, the p-modes manifest their presence at all levels of the outer atmosphere up to the transition region. In the frequency range $v \leq 2 \mathrm{mHz}$, Rutten \& Krijger (2003) identified the signature of gravity waves from TRACE high cadence images at the low chromosphere.

Active region studies with TRACE reveal a whole range of coronal loop oscillations with periods of 2 to $11 \mathrm{~min}$ (e.g. Aschwanden et al. 1999), which are interpreted as kink-mode oscillations induced by flares. These periods are not too far from the periods we report here, but as being active region loop variations they have to be of different physical origin than the quiet Sun internetwork observations of ours. The same holds for the above limb coronal oscillations observed with SUMER, which are interpreted as slow mode loop oscillations, too (e.g. Wang et al. 2003). Furthermore there are reports on propagating slow magneto-acoustic waves e.g. in polar plumes 
(DeForest \& Gurman 1998) or fan-type loops (Berghmans \& Clette 1999). Having periods of about $5 \mathrm{~min}$ and lasting sometimes for hours they are probably driven from the loop foot points (De Moortel et al. 2002). However, none of these phenomena seem to be related to the quiet Sun inter-nerwork regions. In our study we analyzed a SUMER raster including, between others, the spectral line Ne VIII $770 \AA$ emitted at coronal temperatures. It seems that the detected oscillations, in this particular line, above a network cell has not been observed before.

\section{Observations and data reduction}

The SUMER data were obtained on September 22, 1996, when a raster was performed over a quiet Sun area. The field of view was $540^{\prime \prime} \times 300^{\prime \prime}$, centered at solar $(x, y) \sim\left(-200^{\prime \prime}, 430^{\prime \prime}\right)$ and the step size was $3^{\prime \prime}$, for 181 horizontal steps. The recorded spectral lines were Si II $1533 \AA$, C IV $1548 \AA$ and Ne VIII $770 \AA$, the latter one being in second order. The exposure time at each slit position was $150 \mathrm{~s}$, therefore the total duration of the scan was $7 \mathrm{~h}$, from 00:40 to 07:40 UT.

The data treatment is described in detail in Peter (2000). As we are interested only in a rough comparison of the Doppler and radiance maps with the magnetic field structure, we used the closest in time to the scan of this area magnetogram (MDI/SOHO). The selected magnetogram was co-aligned with the SUMER Si II image, as the magnetic field correlates very well with the emissivity from the chromosphere and the low transition region (Fig. 1). We performed a potential field extrapolation in a squared field of view $\left(253^{\prime \prime} \times 253^{\prime \prime}\right)$ centered roughly around a very dark cell, described in the following sections, using the Fourier transform technique (Alissandrakis 1981), taking into account the geometric corrections for a heliocentric angle of $33.8^{\circ}$ from disc center.

\section{A peculiar structure in the coronal Doppler map}

In the Doppler map of Fig. 1, (panel c), the right contour $\left(-100^{\prime \prime}, 500^{\prime \prime}\right)$ outlines a region where one can see vertical stripes, which are aligned exactly with the slit. The exact description of this feature is that pixels aligned along the $Y$ solar axis, parallel to the slit, show variations from red to blue shift for sequential positions along the $X$ axis.

This indicates an oscillation, which cannot be due to an instrumental effect that would affect the data all along the slit. In our case, only a small region of roughly 10 pixels along the slit (Fig. 1) show these variations. Moreover, we took precaution to correct the Doppler shifts due to thermal variations of the SUMER instrument, as done by Peter (1999).

The region where the vertical stripes are seen in Ne VIII Doppler shift, spatially coincides exactly with the inner (dark) part of a chromospheric supergranular cell seen in Si II $1533 \AA$ (panel $\mathrm{b}$, white contour to the right). No oscillation pattern is seen on the brighter part of the network. It is worth noting that the cell where the oscillation is seen corresponds to one of the darkest cells in the low transition region and chromosphere. The same region is also dark in the Ne VIII radiance image (panel a).

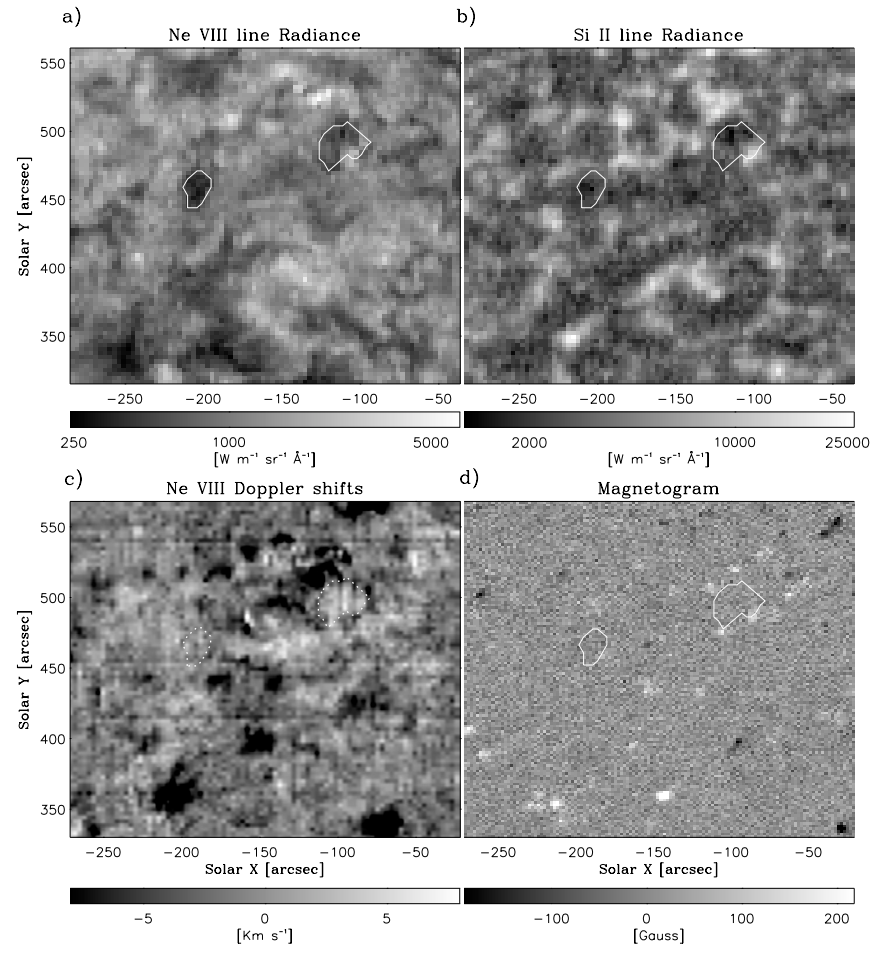

Fig. 1. Part of a SUMER raster showing the line radiance and shift of $\mathrm{Ne}$ VIII (a), c)) formed in the low corona. In the Doppler map dark corresponds to blueshift. Panel b) shows the radiance map in Si II formed in the low chromosphere recorded simultaneously with the Ne VIII maps, while panel d) shows an MDI magnetogram of the same area close in time. The outlines show two very dark network cells.

The stripes cannot be detected as a radiance variation in any of the emission lines (panels a, b of Fig. 1). In the fourth panel of Fig. 1, a MDI magnetogram is co-aligned with the SUMER Si II radiance image. The selected MDI magnetogram was recorded at 04:48 UT, close to the time when SUMER started to raster the oscillating region (05:52 UT). We can see that the Ne VIII oscillation vanishes near the strong magnetic concentrations that surround the cell (panel d).

This finding can be seen in Fig. 2, where we zoom into a part of the raster scan, as vertical stripes in the Ne VIII Doppler map (lower left panel).

It should be stressed that the images in Ne VIII and Si II are exactly co-spatial as they are simultaneously recorded on the detector.

\section{Coronal oscillation above a supergranular cell?}

As only one raster scan of the region of interest is available, we are confronted with a dilemma. Strictly speaking we are not able to distinguish between a temporal and a spatial variation, or a mixture of both. In principle, the variation of the Doppler shift seen as a spatial variation when plotting the raster scan (Fig. 1) could be a spatial variation within the cell, and one might readily start interpreting it in terms of a horizontal standing wave. However, as the structure is exactly aligned to the slit, this seems not very likely. Indeed, the exact alignment with the slit, i.e. the stripes being exactly in the N-S direction, points towards a temporal effect: while scanning over the cell, 


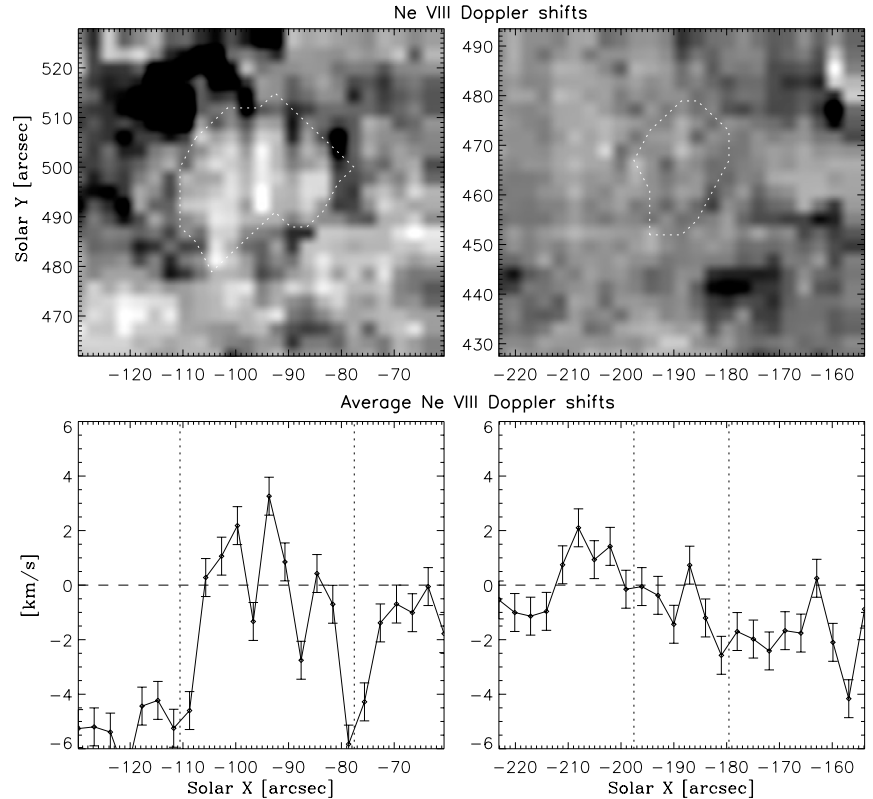

Fig. 2. The top panels show a zoom-in of the Ne VIII Doppler maps of the area around the two network cells outlined in Fig. 1. Vertical stripes are evident in the top left Doppler map. The bottom panels show the variation of the Doppler shift in the scan direction (solar $X$ ) averaged along the slit from solar $Y=490-513^{\prime \prime}($ left panels) and 456-479" (right panels) respectively. Error bars on Doppler shifts are derived from the Gaussian fitting of the individual profiles.

the Doppler shift of the whole cell changes periodically. We will adopt this more likely interpretation for the following discussion of the results.

In Fig. 2 the "oscillating" cell (first collumn) is compared with a non-oscillating one (second collumn). Both cells are very dark in the Si II image. The magnetic field extrapolation showed a stronger magnetic field at the location of the nonoscillating cell, whereas at the oscillating region the field is very weak (Gontikakis et al. 2004). This suggests a higher altitude of plasma $\beta=1$ level for the oscillating cell in contrast to the non-oscillating one.

In Fig. 3, we present the values of radiance, Doppler shifts and line widths, averaged along the $y$ axis over the cell. To compute the time series of panel $\mathrm{j}$ we averaged along half the length of the cell, as only there the oscillations looked coherent and similar to those detected in Ne VIII ones. This way, we converted the spatial scan direction (solar X) into a temporal scale. The oscillation appears in the Doppler shift time series of the Ne VIII line (panel b). We can detect it as three peaks at 12, 18 and $25 \mathrm{~min}$. The same peaks are absent in line radiance and are weak in the line width of Ne VIII (panels a and c). They can be identified in the C IV Doppler shifts (panel e) and in the continuum, but not in the $\mathrm{C}$ IV and $\mathrm{Si}$ II radiance (panels d, g). The continuum, adjacent to the Si II $1533 \AA$ A line, originates from the temperature minimum part of the chromosphere, at about $5700 \mathrm{~K}$.

Let us note that along solar- $X$, i.e. in the scanning direction, we can follow the time variation of plasma features as long as their coherence length scale is larger than a few raster steps. The network cell has a dimension of about 30 to $35 \operatorname{arcsec}$ (cf.
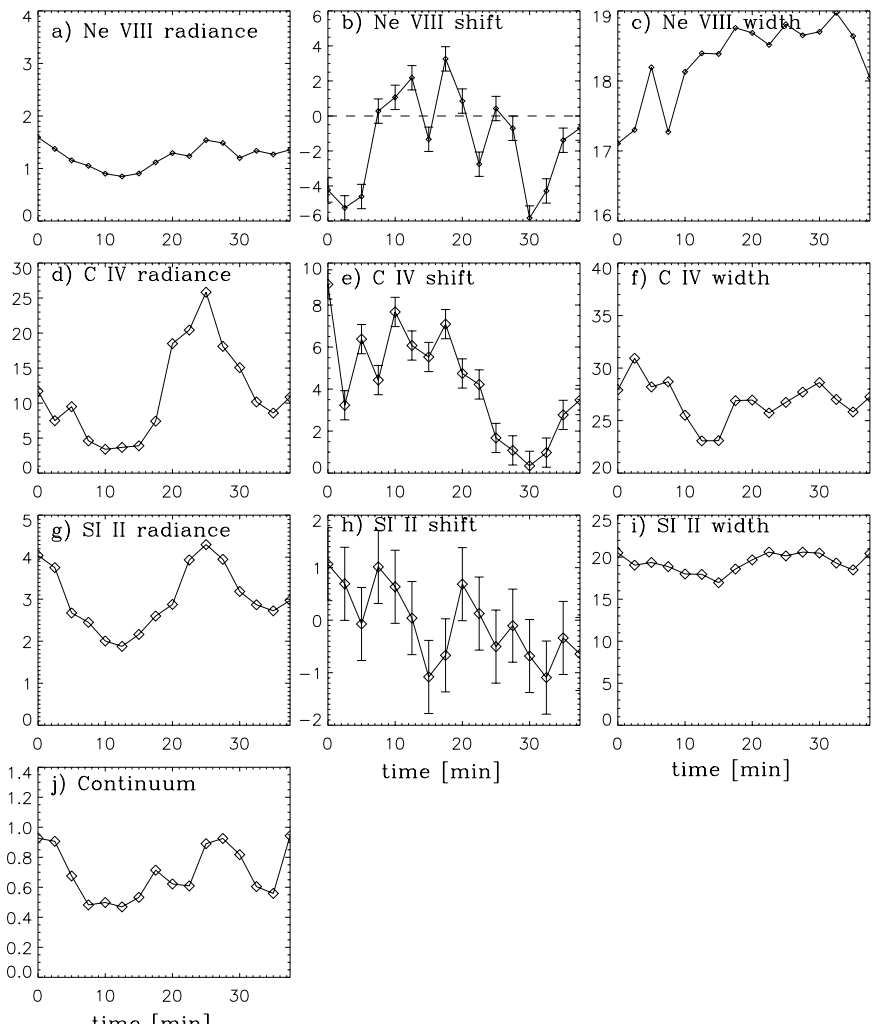

time $[\mathrm{min}]$

time $[\mathrm{min}]$

Fig. 3. Each time series is computed by averaging along the slit, over the oscillating region. First second and third collumns are for radiances, Doppler shifts and Doppler widths respectively. Radiances are in $10^{3} \times \mathrm{W} \mathrm{m}^{-2} \mathrm{sr}^{-1} \AA^{-1}$ and Doppler shifts and widths in $\mathrm{km} \mathrm{s}^{-1}$, positive values correspond to redshifts.

Fig. 2) corresponding to about $24 \mathrm{Mm}$ on the Sun. Thus, the cell covers about 11 raster steps, or $27.5 \mathrm{~min}$ if converted into time needed to scan the cell. Therefore, our data suggest a collective up-and-down oscillation of the whole cell with a period of a bit less than 9 min (or about 3.5 steps).

This is a bit larger than, but roughly comparable to the period of acoustic cutoff that can be computed as $P=\frac{4 \pi c_{\mathrm{s}}}{\gamma g} \simeq$ $7.5 \mathrm{~min}$, where $c_{\mathrm{s}}=16.4 \mathrm{~km} \mathrm{~s}^{-1}$ is the sound speed at the formation temperature of Ne VIII and $g$ is the gravitational acceleration.

\section{Discussion and conclusion}

The response of the quiet solar atmosphere to waves has been quite well studied in the chromosphere up to the transition region, using ground based (e.g. in the $\mathrm{Ca}$ II $\mathrm{K}$ line), as well as space observations (e.g. EUV continua and lines). As mentioned in the introduction, wave propagation has also been widely discussed in active region loops. However, for the low corona in the quiet Sun only few studies exist to investigate oscillatory variations (e.g. Popescu \& Doyle 2004; Morgan et al. 2003). The use of a raster is not appropriate for oscillation studies, as the derived time series are short and undersampled while spatial and temporal variations are mixed. 
The observation we present here is valuable since it gives the opportunity to study a large field of view, which cannot be obtained with a fixed slit observation. We find only one clear signature of an oscillating cell, even though a close inspection of the data reveals some more candidates with less pronounced oscillations. Nevertheless, these oscillating cells may not be such a rare object and further study of more data may bring us closer to an understanding of this phenomenon. The oscillation we observe is mostly seen in the Doppler map of the coronal Ne VIII line. Hence, it wouldn't be detectable in a high cadence imaging instrument like TRACE, neither by CDS due to its low spectral resolution. It is important to note that the oscillation is not seen in the radiance of lines from the transition region and corona.

Higher frequency oscillations were earlier detected in Doppler shift and not in radiance (Judge et al. 1997; Carlsson et al. 1997). It was interpreted in terms of a longitudinal noncompressible wave. In the EUV continuum an oscillatory signature may be present, even though quite weak (cf. Fig. 3, lower right panel). If this is the case, the chromospheric part below the oscillating corona would undergo a compressible fluctuation. This might indicate that the phenomenon is driven from the chromosphere, with a possible mode conversion of the fluctuations at the level of the upper chromosphere from compressible to a non-compressible longitudinal mode (McIntosh et al. 2001; Cally et al. 2001).

Comparing the location of the coronal oscillation with the magnetic field map (and its extrapolation), we find that the oscillation just happens above a region with very weak magnetic field, which would imply a rather high altitude of plasma- $\beta=1$ level. Similar to this argumentation in McIntosh et al. (2001) this would indeed support a mode conversion from the chromosphere into the transition region. However, the oscillation of about 9 min we observe here, does not match the peak of acoustic or gravity wave power (e.g. Rutten \& Krijger 2003).

So far, it is not possible to draw a conclusion on the nature of the oscillatory pattern we presented here. However, this phenomenon may help us understand the connection of the chromosphere with the corona above the so-called non-magnetic inter-network.
Acknowledgements. SUMER is financially supported by DLR, CNES, NASA and ESA PRODEX program. SOHO is a mission of international cooperation between ESA and NASA. C.G. gratefully acknowledges ESA financial support for his participation at the SOHO 15 conference. This program was financed by the Research Committee of the Academy of Athens.

\section{References}

Alissandrakis, C. E. 1981, A\&A, 100, 197

Aschwanden, M. J., Fletcher, L., Schrijver, C. J., \& Alexander, D. 1999, ApJ, 520, 880

Berghmans, D., \& Clette, F. 1999, Sol. Phys., 186, 207

Cally, P. S. 2001, ApJ, 548, 473

Carlsson, M., \& Stein, R. F. 1995, ApJ, 440, L29

Carlsson, M., \& Stein, R. F. 1997, ApJ, 481, 500

Carlsson, M., Judge, P. G., \& Wilhelm, K. 1997, ApJ, 486, 63

DeForest, C. E., \& Gurman, J. B. 1998, ApJ, 501, L217

De Moortel, I., Ireland, J., Hood, A. W., \& Walsh, R. W. 2002, A\&A, 387, L13

Gontikakis, C., Peter H., \& Dara H. C. 2004, in Proc. of the fifteenth SOHO Workshop, Coronal Heating, St Andrews, Scotland 6-9 September 2004, ESA SP-575 December, 2004, ed. R.W. Walsh, J. Ireland, D. Danesy, \& B. Fleck, 131

Gouttebroze, P., Vial, J.-C., Bocchialini, K., Lemaire, P., \& Leibacher, J. W. 1999, Sol. Phys., 184, 253

Judge, P. G., Carlsson, M., \& Wilhelm, K. 1997, ApJ, 490, 198

Judge, P. G., Tarbell, T. D., \& Wilhelm, K. 2001, ApJ, 554, 424

McIntosh, S. W., Bogdan, T. J., Cally, P. S., et al. 2001, ApJ, 548, L237

Morgan, H., Habbal S. R., \& Li, X. 2004, ApJ, 605, 521

Peter, H. 1999, ApJ, 516, 490

Peter, H. 2000, A\&A, 360, 761

Popescu, M., \& Doyle G. 2004, in Proc. of the fifteenth SOHO Workshop, Coronal Heating, St Andrews, Scotland 6-9 September 2004, ESA SP-575 December, 2004, ed. R.W. Walsh, J. Ireland, D. Danesy, B. Fleck, 513

Rutten, R. J., \& Krijger J. M. 2003, A\&A, 407, 735

Wikst $\varnothing 1, \varnothing .$, Hansteen, V. H., \& Carlsson, M. 2000, ApJ, 531, 1150

Wang, T. J., Solanki, S. K., Innes, D. E., Curdt, W., Marsch, E. 2003, A\&A, 402, L17 